\title{
An investigation into thermal necrosis of bone associated with surgical procedures
}

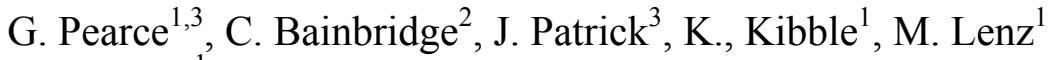 \\ \& G. Jones ${ }^{1}$ \\ ${ }^{1}$ Wolverhampton University, U.K. \\ ${ }^{2}$ Derby Royal Infirmary, U.K. \\ ${ }^{3}$ Robert Jones \& Agnes Hunt Orthopaedic Hospital, Oswestry, U.K.
}

\begin{abstract}
We present the results of an investigation into thermal bone damage or necrosis associated with orthopaedic surgical procedures. Thermal necrosis can lead to infections and pin loosening. It is reported that the critical level at which thermal necrosis appears is when the bone is exposed to a temperature of around $56^{\circ} \mathrm{C}$ over a time of 10 seconds. At this temperature the denaturation point of alkaline phosphatase is reached, which means that the change in properties are directly attributed to the reorientation of collagen molecules and hydroxyapatite.

We present data based on the use of pig bone. No human subjects were used in this study and ethical approval was therefore not necessary.

Drilling was undertaken using an engineering instrument on which the speed may be adjusted. K-wires of varying diameters were used in this drill assembly. Bone sections through drilling points were examined in a scanning electron microscope (SEM). Results are presented in instances where K-wires of different diameters have been inserted by drilling into bone, for different drilling speeds, and for different times taken to drill the K-wire into bones. We present data that indicates that the cooling of bone during surgical procedures may significantly reduce the degree of thermal necrosis arising during orthopaedic surgical procedures.
\end{abstract}

Keywords: bone, trauma, thermal necrosis, injury, $K$-wires.

\section{Introduction}

Bone or osseous tissue is a material that can compete with the best steel reinforced concrete. Roughly one-third of the bone matrix consists of collagen 
fibres, which are tough and flexible. They can easily tolerate stretching, twisting and bending. They are bendable to a certain extent when they are compressed. Although a resilient material, bone is nevertheless susceptible to the effects of heat. Such heating effects can be generated during orthopaedic surgical procedures.

It is well established that thermal necrosis of bone occurs during orthopaedic surgical procedures (Khanna et al. [1]). This may be particularly significant in the case of drilling into areas involving small bone mass, such as drilling K-wire into the phalynx. It would be useful therefore to establish if there are ways of decreasing the degree of thermal necrosis that arises in such procedures.

Khanna et al.[1] investigated the thermal effects of a $\mathrm{K}$-wire fixation on small bones. The aim of their study was to discover the effect of insertion. Their results showed that the time to the peak temperature reduced significantly with the increasing diameter of wire. They also discovered that the tip contour of a $\mathrm{K}$ wire is important as its contouring appears to affect heat dissipation, and that the use of diamond tips produced significantly less heat than the trocar tips of the same diameter.

The authors, however, did not address the question concerning how the time of K-wire insertion and the drilling speed influences the heat generation in bones. Our study addresses this concern, and also looks at the effect of cooling bones prior to drilling on temperature reached.

\section{Materials and methods}

\subsection{The drilling assembly}

Figure 1 shows the arrangement used. The temperature during the K-wire insertion was measured using a thermocouple.

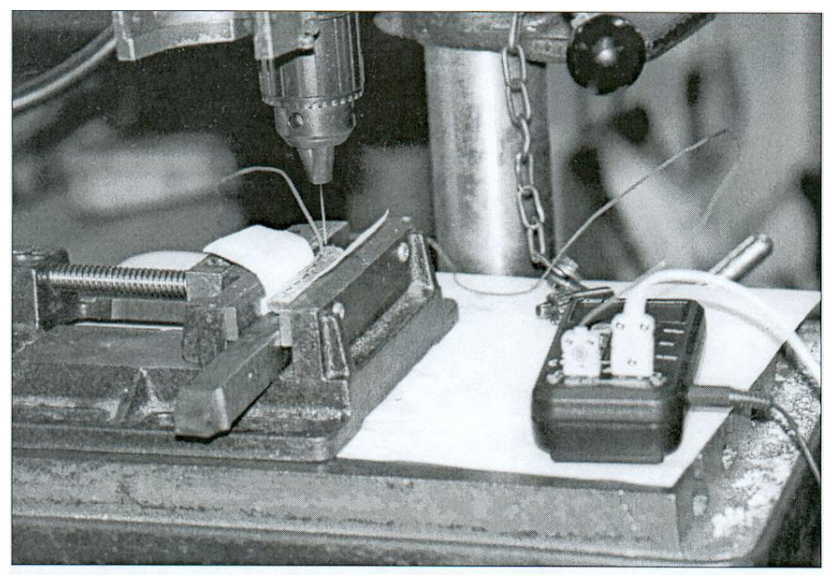

Figure 1: The drilling assembly. 


\subsection{Drilling speed and insertion time}

Since orthopaedic surgery uses a large range of different drilling speeds, the speeds were chosen to be 656, 1180 and $2000 \mathrm{rpm}$. Each time the K-wire was inserted the time of insertion was increased. This factor was shown to significantly change the heat generated. The K-wires were inserted in $10,15,20$, $25,30,35$ and 40 seconds.

\subsection{K-wire diameter}

As we were working principally with hand surgeons for whom heat generation in small bones is a significant problem, the $\mathrm{K}$-wire diameters were chosen to be $0.8 \mathrm{~mm}, 1.0 \mathrm{~mm}$ and $1.6 \mathrm{~mm}$. The trials were carried out with trocar tip K-wires to enable direct comparison with Khanna et al. [1].

\subsection{Bone samples}

In order to achieve a situation very close to real human finger bones during a drilling process, it was necessary to choose bone samples that show the typical outer bone (cortex bone) and the inner bone (marrow cavity). Previous studies (Khanna et al. [1]) had indicated that during the K-wire insertion most of the heat is generated in the cortex portion of bone due to its relatively high density, resulting in a very sharp temperature increase. Khanna et al. [1] also noted that following passage of the K-wire through the first cortex, the temperature curve becomes very flat or decreases over time. This was then followed by an increase of temperature when the wire passed through the second cortex.

Porcine ribs show a well defined bone structure, which made it easy to see when the K-wire passed through the bone. This allowed us to record the exact insertion time and exit time from the bone. Other advantages of these bones were that they were relatively easy to clean, reliable and easy to handle.

Finally, the importance of starting temperature in relation to cooling was investigated. These tests were carried out at a starting temperature of 37 degrees Celsius. The bones were initially cooled in the refrigerator and then drilled at various speeds using a variety of insertion times for the K-wire.

\section{Results}

In this section, we present the results and consider the effects of:

- The temperature change recorded over time for three drilling speeds 656, 1180 and $2000 \mathrm{rpm}$ for 40 seconds of K-wire insertion (figure 2).

- Peak temperature versus insertion time for three different K-wire diameters $0.8 \mathrm{~mm}, 1.0 \mathrm{~mm}$, and $1.6 \mathrm{~mm}$ (figure 3 ).

- Peak temperature versus insertion time for three different drilling speeds: 656, 1180 and 2000 rpm (figure 4).

- Figure 5 shows two temperature curves recorded during a K-wire insertion in a cooled and in a bone at normal body temperature. These 
curves illustrate how the initial bone temperature influences the shape of the recorded temperature-time curve.

- Figures 6,7 and 8 illustrate the results of scanning electron microscopy of the bone to show the effects of different drilling speeds and other variables.

Comparison of different drilling speeds / Bodytemperature / $1.6 \mathrm{~mm} \mathrm{K-wire} \mathrm{/} 40$ sec. Insertion Time

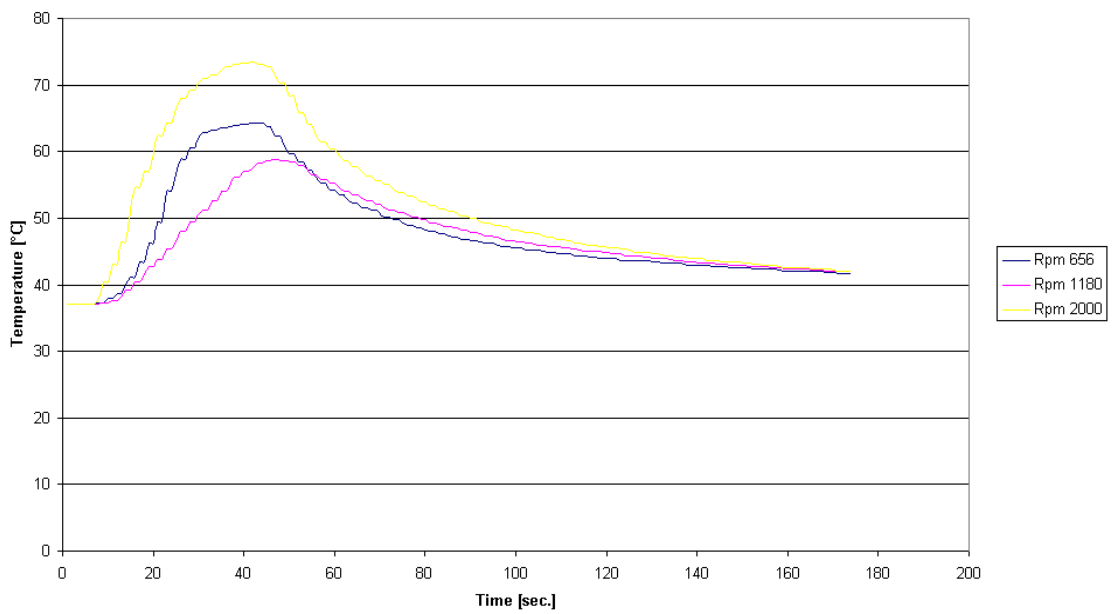

Figure 2: $\quad$ Temperature over time versus drilling speed.

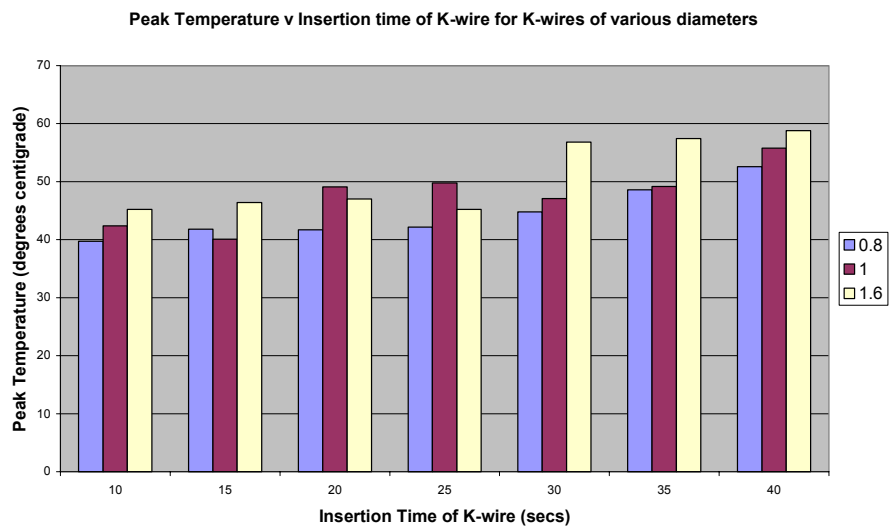

Figure 3: Peak temperature $\mathrm{v}$ insertion time of K-wire for different diameters. 
Figure 2 indicates that the $1.6 \mathrm{~mm} \mathrm{~K}$-wire inserted for 40 seconds at $1180 \mathrm{rpm}$ generated a lower temperature than the same diameter wire inserted at 656 or $2000 \mathrm{rpm}$.

The results in figure 3 show that a $\mathrm{K}$-wire diameter of $0.8 \mathrm{~mm}$ produced the lowest peak temperature, for all insertion times. The $1.6 \mathrm{~mm} \mathrm{~K}$-wire produced the highest peak temperature for all insertion times except for 20 and 25 seconds, when the $1.0 \mathrm{~mm} \mathrm{~K}$-wire produces the highest peak temperature. The total temperature difference between 10 and 40 seconds insertion time for $0.8 \mathrm{~mm} \mathrm{~K}$ wire diameter is just $12.9^{\circ} \mathrm{C}$. For the $1 \mathrm{~mm}$ diameter $\mathrm{K}$-wire, the peak temperatures for 30,35 and 40 seconds are only slightly above the temperatures recorded for $0.8 \mathrm{~mm}$ diameter: the total temperature difference between 10 and 40 seconds insertion time is $13.4^{\circ} \mathrm{C}$. The highest peak temperatures were produced with a K-wire diameter of $1.6 \mathrm{~mm}$, which agrees with the findings of Khanna et al. (1999). The plot shows that for the $1.6 \mathrm{~mm}$ wire, at insertion times between 10 and 25 seconds, the peak temperatures show similar values for insertion times, and there is a sharp temperature increase between 25 and 30 seconds.

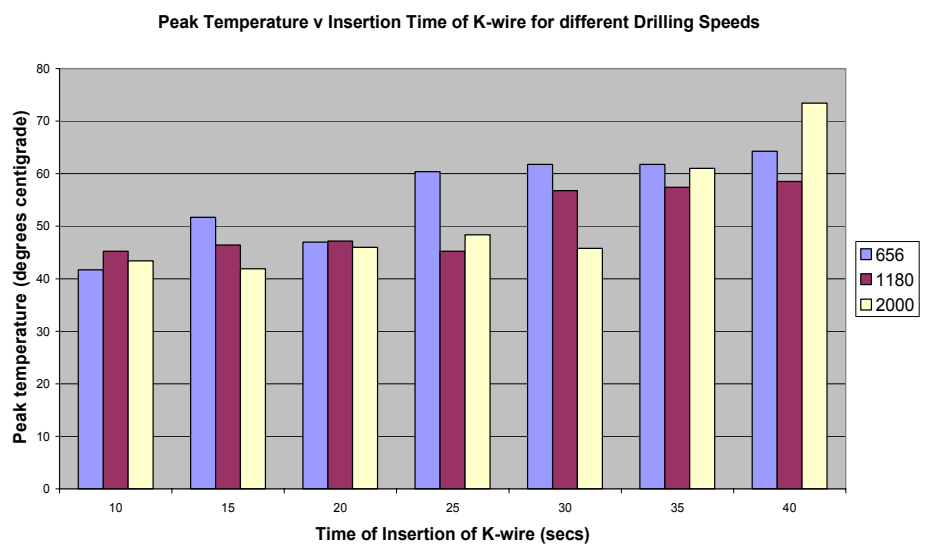

Figure 4: $\quad$ Peak temperature v insertion time; different drilling speeds.

The results presented in figure 4 illustrate the peak temperature achieved for different drilling speeds $\mathrm{v}$ insertion time. The trials were again carried out with a $\mathrm{K}$-wire diameter of $1.6 \mathrm{~mm}$ and drilling speeds of 656, 1180 and $2000 \mathrm{rpm}$. The plot shows that from 25 to 35 seconds, the $0.8 \mathrm{~mm} \mathrm{~K}$-wire generated the highest temperature, although above this the $1.6 \mathrm{~mm}$ wire produced the highest peak temperature.

\subsection{Temperature increase in cool bones}

The results in figure 5 illustrate the influence of cooling on the peak temperature during insertion. In the case of cooled bones, a pork rib was placed for 10 minutes in a fridge. After 10 minutes a K-wire with a diameter of $1.6 \mathrm{~mm}$ was 
inserted with a speed of $1180 \mathrm{rpm}$ in the rib. The insertion times for the K-wire were 10,25 and 40 seconds.

Real Temperatures in cooled bones / Rpm $1180 / 1.6 \mathrm{~mm}$ K-wire diameter

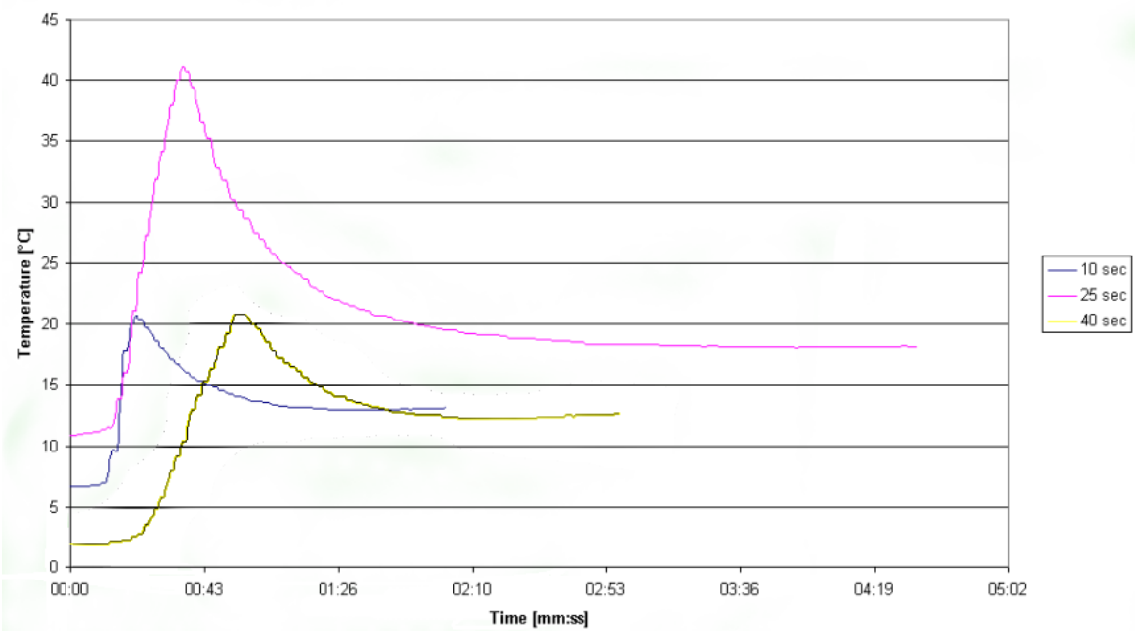

Figure 5: $\quad$ Temp v Time for cooled bone, for different insertion times.

\subsection{Scanning Electron Microscope (SEM) Analyses}

The bones were sectioned and scanned to illustrate the degree of thermal necrosis with various physical parameters applied during drilling. The samples received 40 seconds of $\mathrm{K}$-wire insertion, with different drilling speeds and different $\mathrm{K}$ wire diameters. The analysis focused on the areas of bone where the K-wire passed through the bone, on factors such as the K-wire tip, since the extent of sharpness or blunting of the used wire can be related to its generation of heat. The results are shown in figures 6,7 and 8 .
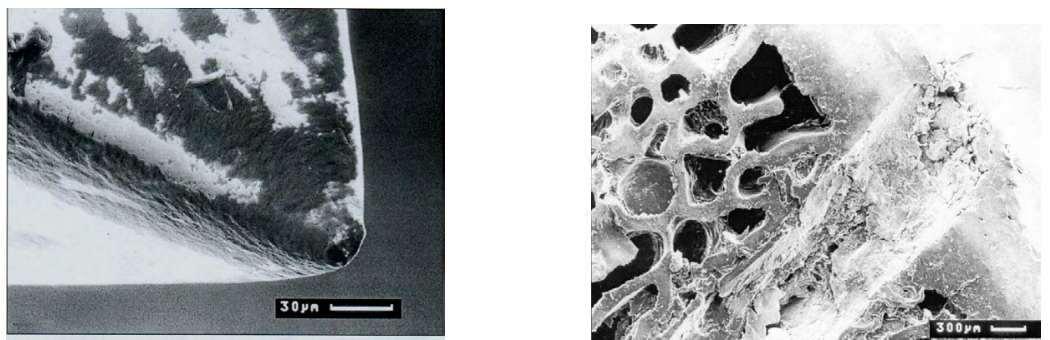

Figure 6: $\quad 1.6 \mathrm{~mm} \mathrm{K-wire;} \mathrm{bone} \mathrm{after} \mathrm{drilling} \mathrm{at} \mathrm{1180rpm.}$ 

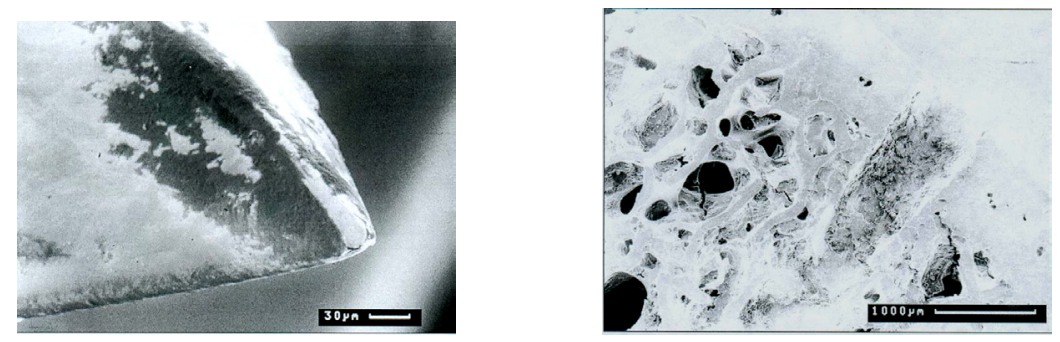

Figure 7: $\quad 1.0 \mathrm{~mm} \mathrm{~K}$-wire; bone after drilling at $1180 \mathrm{rpm}$.
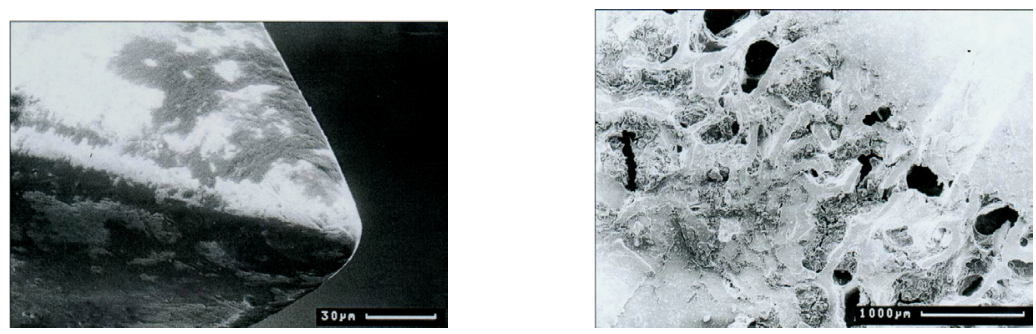

Figure 8: $\quad 0.8 \mathrm{~mm} \mathrm{~K}$-wire; bone after drilling at $1180 \mathrm{rpm}$.

\section{Discussion}

\subsection{Drilling speed}

The three different drilling speeds used for these trials were 656, 1180 and 2000 rpm. Figure 2 shows the temperature curves recorded for the same insertion times. It is noteworthy but not surprising that the lowest drilling speed caused the highest temperature for insertion times of 15 to 35 seconds (figure 4) as more force is likely to be used to insert the wire at lower speeds. The temperature increase for $656 \mathrm{rpm}$ is approximately twice the increase recorded for $2000 \mathrm{rpm}$. The drilling speed $1180 \mathrm{rpm}$ appears to be the optimum for keeping the temperature low under both speeds of insertion.

Generally the higher the total peak temperature, the sharper the temperature increase at the beginning of the insertion. It might be expected that the higher the drilling speed, the higher the total peak temperature. This unexpected behaviour may either be explained by a different thickness in bone structure of the used samples, or the lower the drill speed, more pressure was required to insert the Kwire, resulting in a higher generation of heat. This will be the subject of further investigations. 
According to these results, a speed of $1180 \mathrm{rpm}$ should be considered for Kwire insertion, which is also supported by the results shown in Figure 4. Figure 4 shows the absolute peak temperatures recorded for drilling speeds of 656,1180 and $2000 \mathrm{rpm}$, for insertion times between 10 and 40 seconds. It shows that a speed of $1180 \mathrm{rpm}$ has the most constant values, although between 25 and 30 seconds insertion time a sharp temperature increase was recorded. The total temperature difference for this speed between 10 and 40 seconds insertion time is only $13.6^{\circ} \mathrm{C}$. A speed of $656 \mathrm{rpm}$ shows the most fluctuating values. This speed also shows a sharp increase in temperature between 20 and 25 seconds insertion time. Between an insertion time of 25 and 40 seconds the peak temperature only increased slightly by $3.9^{\circ} \mathrm{C}$. The total temperature difference at this speed is $22.6^{\circ} \mathrm{C}$. However, a speed of $2000 \mathrm{rpm}$ shows interesting behaviour. Prior to an insertion time of 30 seconds, all peak temperatures are below $50^{\circ} \mathrm{C}$, and they only fluctuate between $6.5^{\circ} \mathrm{C}$. However, between 30 seconds and 40 seconds insertion time, the peak temperatures increase very sharply. The temperature difference between an insertion time of 30 seconds and 40 seconds is $27.6^{\circ} \mathrm{C}$, and the total temperature difference between 10 seconds and 40 seconds is $31.5^{\circ} \mathrm{C}$.

The analyses have shown that, in general the insertion time should be kept as short as possible for all speeds tested. For insertion times up to 30 seconds a speed of $2000 \mathrm{rpm}$ should be considered, when all the peak temperatures stayed below $56^{\circ} \mathrm{C}$. For insertion times of 35 to 40 seconds, a speed of $1180 \mathrm{rpm}$ should be used.

\subsection{K-wire diameter and tip sharpness}

The analyses of the recorded temperature curves have already shown that generally, the larger the K-wire diameter, the more heat is generated.

Results indicate that a K-wire with $1.6 \mathrm{~mm}$ diameter, which was inserted in 40 seconds at a drilling speed of $1180 \mathrm{rpm}$ produced at $58.8^{\circ} \mathrm{C}$ the highest peak temperature compared to the other diameters. As the K-wire does not transport the bone away, (due to the absence of a screw thread) but only compresses it, the density increases, which again leads to more heat generation.. See figure 6 .

One possible reason for the high peak temperature may be found in considering the tip of the K-wire. The results also indicate that the sharpness of the drilling wire can be directly related to its generation of heat. Figure 6,7 and 8 also show the effects on bone structure that resulted during the drilling trials. The change in grey scaling indicates the degree of thermal necrosis. The appearances illustrated show that when the wire passed through the bone, the intricate network structure in the medullary cavity becomes damaged by compression.

A K-wire diameter of $0.8 \mathrm{~mm}$ produced the lowest temperature for the analysed trial. It was inserted within 40 seconds with a drilling speed of 1180 rpm. Figures 6, 7 and 8 show the image of the K-wire tip. It can be seen in figure 8 that the tip and the comers of the wire still seem to be sharp. This could explain the relatively low generation of heat of only $52.5^{\circ} \mathrm{C}$. The analysis of the bone structure showed that this K-wire caused less damage and compression of bone. 
As the compressed area, and that with the density of bone increases with K-wire diameter, this could explain the low peak temperature recorded.

A comparison of the temperature curves recorded for different drilling speeds revealed that a speed of $1180 \mathrm{rpm}$ generated the lowest peak temperature, followed by speeds of 656 and $2000 \mathrm{rpm}$. A drilling speed of $1180 \mathrm{rpm}$ generated the lowest heat for most of the trials undertaken, and figures 6,7 and 8 show the SEM images of the K-wires. The comers and the tip show little evidence of wear, although both still appear to be quite sharp. The peak temperature for this $\mathrm{K}$-wire was recorded to be $58.8^{\circ} \mathrm{C}$. Figures 6,7 and 8 also show the bone structure resulting from this. Again damage in the network structure caused by compression is present, and also changes in bone structure itself occurred.

In summary, the drilling speed, time of insertion, sharpness and contour of the $\mathrm{K}$-wire, in addition to the bone quality and initial temperature, all appear to be factors that influence thermal necrosis in bone. These factors are worthy of further investigation, and should be considered in unity in orthopaedic surgical procedures, especially in the drilling of small volumes of bone (e.g. phalanges).

\section{Conclusion}

This study investigated the influence of several parameters i.e. K-wire diameter, drilling speed, insertion speed and the effect of cooling on the generation of heat during a K-wire insertion. To achieve this aim commonly available K-wires made of stainless steel were used, of different diameters of $0.8 \mathrm{~mm}, 1.0 \mathrm{~mm}$ and $1.6 \mathrm{~mm}$. The effect of the insertion time was investigated. Each K-wire was drilled between 10 seconds and 40 seconds through the test sample. In addition three different drilling speeds were used, namely of 656, 1180 and $2000 \mathrm{rpm}$. Finally, the effect of cooling on the total temperature increase was analysed.

The results have shown that:

- the insertion time is one of the main parameters that influence the heat generation during a drilling process.

- $\quad$ a trend can be seen, namely the longer the time the insertion takes place over the higher the total peak temperature, irrespective of drilling speed or K-wire diameter.

- trials undertaken with cooled bones showed that the temperature increase was not only related to the insertion time of the K-wire, but also to the starting temperature of bone for insertion.

Generally speaking, it can be concluded that:

- data shows a trend in temperature increase with an increase in K-wire diameter, in line with the findings of Khanna et al. (1999).

- $\quad$ analyses obtained from the SEM have shown that indeed the sharpness of the tip of a K-wire has an influence on the heat generation during insertion. For very high peak temperatures and large diameters the Kwire trocar tip seemed to become damaged during drilling.

- The SEM examination also showed that a smaller diameter K-wire produces less damage to the surrounding bone tissue. 
- All the bones analysed showed damage in network structure caused by a compression in the drilling process. The relatively low generation of heat for smaller diameters can be explained.

It should be noted that the bone stock and structure itself, may influence results, and this is worthy of further study.

The results from the data recorded for different drilling speeds also showed interesting behaviour. It was expected that the heat generation increases with drilling speed, and it was surprising that the drilling speed of $1180 \mathrm{rpm}$ generated the lowest temperatures for the higher insertion times compared.

In summary, the results presented in this study are not only worthy of further research, but suggest that such factors as drilling speed, diameter of K-wire, and initial bone temperature (prior to the start of drilling) may have a profound impact on the extent of the thermal necrosis of bone arising during the course of drilling during orthopaedic surgical procedures.

One other potential area for extension of this study would be to try to define the relationship between the various parameters, such as type of metal used, volume of bone removed, pressure on the $\mathrm{K}$-wire, the $\mathrm{K}$-wire diameter and necrosis generated. This would enable mathematical modelling of the process and hence make the procedure of inserting of the K-wires safer.

\section{References}

[1] Khanna, A., Plessas, S.J., Barrett, P., Bainbridge, L.C., (1999). Journal of Handsurgery (British and European Volume) 24B:3 355-357.

[2] Baumgart, F., Kohler, G. and Ochsner, P., (1998) The physics of heat generation during reaming of the medullary cavity Injury Vol. 29, Suppl. No.2, pp. S-BI1-S-B25, 1998

[3] Namba, R., Kabo, J.M., Meals, R.A., (1987) Biomedical Effects of Point Configuration in Kirschner-wire Fixation Clinical Orthopaedics and Related Research (Number 214, January 1987). 